\title{
Process of Psychological Growth Associated with the Experience of the Yips Using the Trajectory Equifinality Model
}

\author{
Matsuda Kojiro $^{1 *}$, Susaki Yasuo ${ }^{2}$, Mukai Kosuke ${ }^{3}$, Yoshio Sugiyama ${ }^{4}$ \\ ${ }^{1}$ Faculty of Rehabilitation, Kyushu Nutrition Welfare University, Kitakyushu, Japan \\ ${ }^{2}$ Faculty of Education, Shimane University, Matsue, Japan \\ ${ }^{3}$ Office for Students with Disabilities, Osaka Ohtani University, Tondabayashi, Japan \\ ${ }^{4}$ Factory of Human-Environment Studies, Kyushu University, Fukuoka, Japan \\ Email: *kojiromatsuda612@gmail.com
}

How to cite this paper: Kojiro, M., Yasuo, S., Kosuke, M., \& Sugiyama, Y. (2019). Process of Psychological Growth Associated with the Experience of the Yips Using the Trajectory Equifinality Model. Psychology, 10, 1881-1907.

https://doi.org/10.4236/psych.2019.1014122

Received: October 13, 2019

Accepted: November 12, 2019

Published: November 15, 2019

Copyright (c) 2019 by author(s) and Scientific Research Publishing Inc. This work is licensed under the Creative Commons Attribution International License (CC BY 4.0).

http://creativecommons.org/licenses/by/4.0/

\begin{abstract}
The purpose of this study was to explore the process of positive psychological changes (i.e., psychological growth) with the experience of the yips using qualitative research methods, focusing on positive aspects among the psychological changes with the experience of the yips. We interviewed one baseball player who had experienced the yips in the past and gained data on the psychological growth associated with the yips experience and what is believed to be involved in it. We then used Trajectory Equifinality Model to analyze the data obtained from the interviews. The analysis yielded four categories of psychological growth with the experience of the yips. These results suggest that "psychological conflict and struggle following the experience of the yips", "intentional and active meaning-making with regard to the yips and throwing", "social support" and "overcoming the yips" play important roles in their psychological growth.
\end{abstract}

\section{Keywords}

Posttraumatic Growth (PTG), Negative Experiences, Crisis, Turning Point

\section{Introduction}

The yips is a disorder characterized by involuntary movements occurring during the performance of automated motor skills requiring fine control, such as those executed by baseball players, golfers, bowlers (in cricket), table tennis players, tennis players, darts players, and many other athletes who are required to carry out a target task (Adler, 2012; Bawden \& Maynard, 2001; Clarke et al., 2015; Le 
Floch et al., 2010; McDaniel et al., 1989). In Japan, research on the yips among baseball players has been conducted for a long time (Iwata \& Hasegawa, 1981; Kagawa, 2013; Nakagomi, 1987). For example, Mukai (2016) conducted interviews in a survey of university baseball players who had been afflicted with the yips and pointed out that improvement in or prolongation of the yips tends to be influenced by how affected athletes perceive and endure their experiences of the yips. Symptoms of the yips among baseball players include "even though it is a short distance, throwing a band or throwing it in a place that a partner cannot catch" (Kagawa \& Fukae, 2013: p. 440); additionally, players feel that their arms shrink or they cite weakness in their arms for certain distances or situations (Mukai \& Koga, 2017).

Other studies describe the yips as a movement disorder that appears during the execution of an athlete's motor skills (Dhungana \& Jankovic, 2013; Smith et al., 2003); such disorders faced by athletes cause a great deal of psychological damage. For instance, Aoki and Matsumoto (1999) pointed out the possibility of "disgust and aversion for sport[s] activities" (p. 9) or "deviant behavior" (p. 9). The authors also mentioned the retirement of athletes as a negative influence of movement disorders experienced by athletes. Findings of other studies indicate that the experience of the yips has caused negative psychological changes among athletes, such as anticipatory anxiety, fear, irritation, and as a worst case scenario, escape or retirement from one's sport (Bawden \& Maynard, 2001; Nakagomi, 2004; Philippen \& Lobinger, 2012; Smith et al., 2000).

Thus, the negative influences of the yips on athletes, including negative psychological changes in athletes who have experienced the affliction, have been proven. However, there has been little research on the positive aspects associated with athletes' experiences related to the yips and the subsequent psychological changes. To accurately understand the various experiences encountered by athletes in their sports, there is a need to clarify both the negative and positive aspects of the issues they face (e.g., Wadey et al., 2011).

Incidentally, in the areas of positive psychology and clinical psychology, it has been reported for a long time that negative experiences possibly trigger psychological growth (Affleck et al., 1985; Thompson, 1985). Schaefer and Moos (1992) reviewed a number of previous studies in "Life Crises and Personal Growth, Yasuda", their chapter from the book, Personal coping: Theory, research, and application. They argue that a negative experience triggers psychological growth. One of the representative concepts for explaining psychological growth that accompanies a negative experience is posttraumatic growth (hereinafter, "PTG"). Evidence for the validity of this concept is psychological growth experienced as a result of such traumatic events as natural disasters (e.g., typhoons, earthquakes), illness, injury, and death of a family member. Subsequent psychological conflict and struggle have been reported (Taku, 2016). In addition, Taku (2016) has suggested that traumatic events associated with PTG are not only events related to life and death, but also events that are accompanied by some kind of stress, such 
as failure to pass an exam or immigration of cohabitants (p. 3). Furthermore, to explain psychological growth resulting from a negative experience, as well as PTG, there are many concepts such as "finding benefits" (Jansen et al., 2011), "stress-related growth" (Park et al., 1996), and "adversarial growth" (Linley \& Joseph, 2004). In recent studies in the area of sport psychology, the negative experiences (e.g., sports injuries, conflicts with coaches and teammates, failures in serious situations that trigger trauma) that athletes face in the sports scene are beginning to be pointed out as triggers for psychological growth as an athlete (Tamminen et al., 2013; Tamminen \& Neely, 2016; Wadey et al., 2013).

In addition to these earlier studies, Matsuda et al. (2018) speculated that the experience of the yips is not only a negative influence psychologically, but there is the possibility that it may bring about psychological growth for an athlete. They conducted research, dividing 416 baseball players into three groups: the non-yips group (had never experienced the yips), the yips group for whom symptoms of the yips continued to appear, and the group that overcame the yips. The authors examined whether there was a difference in the degree of afflicted athletes' psychological growth.

Furthermore, to measure the degree of psychological growth for athletes, we adopted five subscales (i.e., A scale) for measuring their mental maturity (hereinafter, "MMAA"), which were created by Sugiura (2001). As a result, on three subscales of MMAA, the group that overcame the yips scored significantly higher than the non-yips group. In addition, the three subscales recognized here are said to have an "easy-to-improve" feature in terms of the negative experiences faced by athletes (Sugiura, 2001). On the other hand, scores were not significantly higher for the yips group on any subscale compared to those for the other groups. Matsuda et al. (2018) correlated these findings with the findings of a previous study and pointed out that the psychological growth observed in people who have experienced the yips is not merely because of the experience of the yips, as overcoming the yips also plays an important role in growth.

However, in the study by Matsuda et al. (2018), only the relevance between the experience of the yips, overcoming the affliction, and psychological growth as an athlete is demonstrated; the kind of pathway that brings about these associations (e.g., the process by which an athlete experiences the yips and overcomes the affliction, then demonstrates psychological growth) has not been verified. By clarifying the process just described, this study can help athletes who have experienced the yips or an athlete's coach to reconsider how they recognize and cope with the yips.

To clarify the process-from experiencing the yips to recognizing psychological growth as an athlete-, we considered that a research method to retrospectively examine psychological changes since being afflicted with the yips or overcoming the disorder, as well as the factors related to the changes occurring with the passage of time, would be useful. Therefore, in the present study, we conducted an analysis based on the Trajectory Equifinality Model (hereinafter, 
“TEM") (Sato, 2006; Sato et al., 2006; Sato et al., 2007; Sato \& Tanimura, 2016; Sato \& Valsiner, 2010; Valsiner, 2011; Watzlawik \& Kullasepp, 2016), a methodological framework for observing the temporal changes in human growth socially and culturally. "Equifinaly" is the idea that people have more than one path to reach a goal, or that they can follow different paths but achieve similar results (Lyra et al., 2018; Valsiner \& Sato, 2006; Zittoun \& Valsiner, 2016). Meanwhile, "Trajectory" is a concept introduced by Valsiner (2000) in the field of developmental psychology and cultural psychology to show the multi-linearity of developmental pathways of people and when applied to the idea of Equifinaly, it is a concept showing the diversity of routes to a goal (Lyra et al., 2018).

The TEM has been used in various areas, including the process by which ability is developed for performance (Tominaga et al., 2015), the process leading to athletes becoming Olympians (Hayashi \& Tsuchiya, 2012), life course decision-making processes of individuals with mental retardation (Kasada, 2013), and the progression of psychological changes in unmarried women who experience abortion (Yasuda et al., 2008). The main feature of the TEM is not to abstract time. Time mentioned here is not that measured by a clock; rather, it is sequential time (passage of external time) (Tominaga et al., 2015). The TEM is based on the concept of "irreversible time" (Sato, 2009: p. 5), indicating sequential time (e.g., sustained time that does not backtrack). Moreover, the TEM is based on the following idea: individual actions and choices accompanying a certain experience or process of psychological changes-even if they follow different paths - have convergent or equal arrival points-hence, the "Equifinality Point" (hereinafter, the "EFP”) (Sato et al., 2006). Furthermore, the EFP is regarded as "a focal point in research, set by researchers" (Kasada, 2013: p. 231). Therefore, in the present study, overcoming the yips and psychological growth as an athlete were the basis for the EFP.

By placing emphasis on the idea of irreversible time and identifying the EFP as overcoming the yips and experiencing psychological growth as an athlete, it is possible to visualize the path for various psychological changes up to that point and certain factors related to changes with the passage of time. Furthermore, Ueda (2013) mentioned that the TEM can clearly show the process by which individuals make selections (branch points), follow various paths (multiple paths), and reach some final state (e.g., EFP) while mutually negotiating with the outside world.

Findings of this study are expected to not only clarify psychological changes of athletes who experienced the yips, but also clarify the kind of psychological changes that are occurring in mutual negotiations with the outside world. From the above, we aimed to clarify in this study the path and process of psychological growth in an athlete that accompanies an experience of the yips and overcoming it; in addition, we identified some factors that could trigger such psychological growth by utilizing the TEM to explore this process. Furthermore, psychological 
growth as an athlete used in the present study conforms to the definition defined by Sugiura (2004a: p. 24): "positive changes in psychological aspects that are necessary for athletes to do sports." Regarding the yips, we used the following definition (with slight modifications) by McDaniel et al. (1989) for this study: "movement disorders composed of involuntary movements occurring during the execution of automated motor skills that require fine control."

\section{Method}

\subsection{Participants}

To extract collaborators for the present study, we adopted Historically Structured Sampling (hereinafter, "HSS"), which is inseparably related to the TEM. Concerning the HSS, Mukai (2016) pointed to the "EFP according to the purpose of the study and selecting [an] experienced person of that EFP as a survey participant." Therefore, sampling was carried out based on the EFP (i.e., overcoming the yips and psychological growth as an athlete) that was established according to the purpose of this study.

As a cooperator in the survey, we selected a university baseball player (hereinafter, "Participant A"). This 19-year-old male recognized that he had experienced the yips but had already overcome the disorder and acknowledged that psychological growth as an athlete was promoted through those experiences. As the specific extraction method, the following five inclusionary criteria based on previous studies (Bennett et al., 2015; Mukai \& Koga, 2017; Sachdev, 1992; Smith et al., 2000) were used to select collaborators suitable for the purpose of this study. Then, we selected Participant A as a collaborator of the survey, which was confirmed to satisfy all five criteria. Confirmation of these inclusion criteria was incidentally confirmed via e-mail and telephone and exchanged on the day of the survey prior to its administration:

1) Those who have experienced obvious abnormalities, such as continuing to throw a wild ball that the opponent cannot catch or continuing to bounce the ball in front of themselves

2) Those who have been able to reliably perform the behavior that the symptom of the yips appeared

3) Those who have experienced symptoms as not temporary but continuously appearing for more than a month

4) Those who recognized that they have returned completely to the state before symptoms appeared

5) Those who recognize that psychological growth as an athlete was fostered by his or her experience of the yips

\subsection{Data Collection}

We conducted an episode interview with Participant A based on the following interview guide. The episode interview attempt to exploit the advantages of both a narrative interview and a semi-structured interview are a way to approach spe- 
cific experiences in interviews related to research subjects rather than narrative about life as a whole (Flick, 2007).

Furthermore, we asked Participant A, "Please answer this time with awareness of the passage of time and causal relationship." The first author asked another question related to the part not mentioned in detail and the part needing further explanation. This approach was repeated, and the interview was performed face-to-face and was lasted two hours (until each story could be fully understood). The entire content of the interview was recorded with an IC recorder and field notes. The survey was conducted in a room at the university that Participant A belongs.

\subsection{Interview Guide}

The present study was conducted based on the following items from the interview guide:

1) Profile (age, university, grade, and sport experience other than baseball, defensive position, living environment, and dietary habits)

2) Career/awards in sports (the reason he started baseball, Award received between elementary school, junior high school, and high school)

3) Regarding baseball (Reason for playing baseball, feelings/passion/thoughts toward Baseball, Priority of Baseball in life)

4) Experience of the yips (symptoms, scene in which the symptoms appeared, coutermeasures, overcoming the affliction)

5) Relationships with coaches, teammates, and other people (the person who he doesn't like, the person who he likes, the relationships before experiencing the yips, and relationships after experiencing the yips)

6) Wanting to quit baseball because of the occurrence of the yips (the experience of having "I wanted to quit baseball" thoughts in the past, the specific reason why he wanted to quit baseball, the reason why he did not quit baseball)

7) Experiences of suffering from symptoms of the yips and simultaneous changes in views and attitude toward sports and oneself (the experience of conflicts due to the experience of the yips, the changes in the thought and attitude toward sports through the experience of yips, the changes in views of themselves through the experience of the yips)

8) Physical or technical changes in the competitive situations (including practice) since experiencing the yips

9) Future plans (future dream, job, Concerning the Continuation of Baseball in the Future, the changes in the thought for their future through the experience of yips)

\subsection{Data Analysis}

As an analytical method in the present study, the TEM was adopted. By using it to model Participant A's specific experience of the yips and the process that led to psychological growth as an athlete (i.e., time series), we expected to deepen 
our understanding of the series of events and changes he experienced.

The main concepts of the TEM used in the present study are provided below. First, regarding the EFP, it was as described above. Moreover, the concept defined as "something assumed to be an event completely contrary to EFP or a complement to actions and choices focused as EFP" (Yasuda \& Sato, 2012: p. 34) is referred to as the "Polarized EFP" (hereinafter, "P-EFP"). The inclusion of this concept is recommended when conducting TEM analyses as it "prevents valuation of EFP and makes it possible to draw a balanced TEM graph" (Wada, 2016: p. 49) by depicting hypothetical events and paths in cases that fall short of EFP. Additionally, it helps resolve one of the weaknesses of qualitative studies-namely, the relatively small number of participants, when compared to quantitative studies that employ survey questionnaires, for example (Sato, 2009). Moreover, the P-EFP sometimes depicts events not obtained via a survey. Next, many people should pass (Kawai et al., 2016: p. 4) the Obligatory Passage Point (hereinafter, "OPP”). However, since the present study is a single case study, the combination of the results of Participant A's narrative and previous studies and set points must be experienced theoretically or customarily as the OPP. Additionally, there is the concept of social guidance (hereinafter, "SG"), defined as "one that acts to support a step toward EFP" (Wada, 2016: p. 50).

Incidentally, the Grounded Theory approach (hereinafter, "GTA") invented by Glaser and Strauss (1967) has been widely applied to analyze quality. The main feature of the GTA is that it can capture similar events, experiences, and interactions repeatedly (Harada et al., 2009). On the other hand, regarding the TEM, it is that by "expressing the personal experience (from the left to the right picture in the figure) with a flow of irreversible time, it is not suitable for expressing the cyclic process of returning" (Hanashima, 2018: p. 29).

In the process of coping with the yips, symptoms are repetitive and include turning around, deteriorating, advancing, and retreating many times (Marquardt, 2009). Furthermore, depending on the circumstances, the situations experienced by an athlete coping with the yips may not necessarily occur sequentially. In the TEM graph, there is a constraint on irreversible time from left to right, so it is difficult to fully express such situations. Therefore, in the present study, the GTA paradigm was partially incorporated into the TEM process with reference to Hanashima (2018), and a more accurate process figure was created. Concretely, some categories in the process of coping with the yips was included in a circle together, and a circulating process was illustrated partially.

Additionally, the specific analytical procedure of present study is as follows. the specific analytical procedure was based on studies from Hayashi and Tsuchiya (2012) and Ohkubo (2001). First, transcriptions were produced from the data captured on the IC recorder; additionally, a person's name was replaced with " $X$." Then, the contents of the transcription were divided into sections based on meaning, and headings that briefly described the content of each were applied. Moreover, in each heading, we summarized contents with similar cha- 
racteristics. Then, from the summarized headlines, narratives related to the following contents were extracted: experience of the yips, overcoming the yips, challenges when overcoming the yips, psychological changes accompanying the experience of the yips (e.g., "Prior to experiencing the yips, I thought that ..., but the experience of the yips made me [...]." "Because of experiencing the yips, I grew in the sense of [...]") and what seemed to be involved in its psychological changes. Then, the contents were arranged along the flow of time, and a TEM graph was created. Ookubo (2008) pointed out that by arranging the contents of a narrative according to the context, the story's properties would become visible.

To enhance the validity and rigor of the analysis, we proceeded with all stages of the analysis with one of the collaborative authors; if the interpretations of both did not match, we repeatedly discussed our views with each other until we arrived at a satisfactory explanation. By collaborating with others, we made it possible to reduce the risk of bias in a lead author's arbitrariness. One of the collaborative authors is a clinical psychologist and has experience conducting psychological counselling with athletes and others with psychological problems. Moreover, he has many research achievements in qualitative research and research related to the yips. Additionally, to increase reliability, we provided detailed descriptions of Participant A's various viewpoints and psychological changes to suggest how to deal with the yips comprehensively. Research based on a single case example (as in the present study) makes it possible to create a more substantial description than that in an investigation of many subjects for the purpose of generalization (Yamada, 2007).

Finally, as a representation of the TEM graph, each headline is surrounded by a solid line; the OPP is surrounded by double lines, and the EFP is enclosed within thick lines. In addition, the P-EFP was enclosed within a dotted line. Regarding the arrows, the contents resulting from the interview with Participant $\mathrm{A}$ are indicated by solid lines, and the paths that could not be mentioned but which were considered to be theoretically possible are indicated by dotted lines. Furthermore, the upward arrow on the vertical axis indicates SG, and-to represent irreversible time-an arrow extends horizontally from left to right.

\subsection{Ethical Consideration}

We conducted research for this study with the approval of the Ethics Committee of Human-Environment Studies, Kyushu University, Japan.

\section{Results}

As mentioned previously, we set overcoming the yips and psychological growth as an athlete as the EFP and we visualized the psychological changes taking place on the path toward that EFP and the factors related to psychological changes (Figure 1). Furthermore, since it was the main purpose of the present study to consider the process of psychological growth accompanying the yips, content provided in the interview regarding the participant's condition before experiencing 


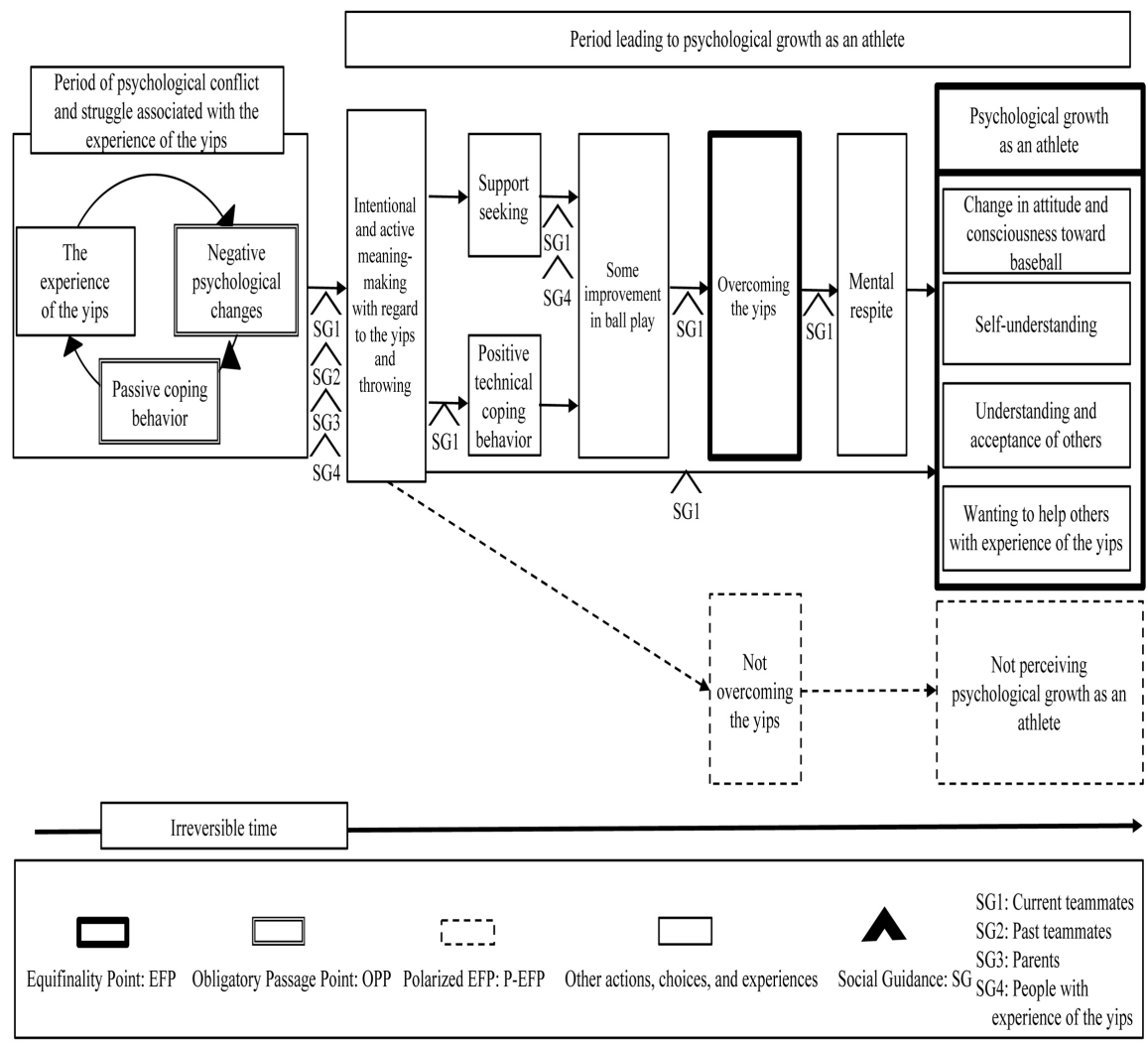

Figure 1. TEM graph of process of psychological growth associated with the experience of the yips.

the yips was excluded from the analysis and TEM graph.

We show simple information from Participant A's interview, then we explain TEM concepts relevant to the present study. Furthermore, fragmented experiences, OPP, EFP, and P-EFP are indicated with $<>$, and SG is indicated with [ ].

In the interview with Participant $\mathrm{A}$, he explained that he began playing baseball in the third grade (elementary school), and he competed for 10 years. His positions were pitcher and infielder. In addition, Participant A has competed at a high level, with a past victory in a regional tournament and experience participating in Koshien. ${ }^{*}$ The university that he attended has recruited many professional baseball players so far; in fact, it is regarded as having the top baseball team among Japanese universities. Regarding the yips, symptoms began to appear immediately after he joined the university's baseball team, and they appeared continuously for about four months. Specifically, his throwing motion was affected, and from the narrative, we hear that he was very confident about throwing originally:

I was, like, condescending (to teammates)... I honestly think that was the case sometimes.... For example, I'd say, "It's obvious you throw against the chest when playing catch," "If you can't throw, you're out," "You suck"stuff like that." Next, when asked about causes of the yips, he said, "I didn't 
intend to do baseball after retiring from high school...; I just played around and did other things like soccer.... And then, I hadn't held a baseball in two or three months.... I played a bit of catch before starting uni ... I thought maybe doing this amount was about right.

He identified factors like neglecting to practice pitching and throwing as well as thinking lightly of it. Participant A's narrative concerning his experience of the yips and the headings briefly showing its contents are shown in Table 1.

As described above, <overcoming the yips $>$ and $<$ psychological growth as an athlete $>$ (Table 2) were established as the EFP according to the purpose of the present study. At the same time, <not overcoming the yips $>$ and $<$ not perceiving psychological growth as an athlete $>$ were established as the P-EFP-that is, events contrary or complementary to the two EFPs. Participant A experienced various $<$ negative psychological changes $>$ immediately after his affliction with the yips for the first time; what subsequently followed was a $<$ passive coping behavior>. These two points were thought to be points that many people who experienced the yips passed through or felt obliged to pass through; hence, the OPP was established.

Finally, we set "social support" as SG, which is considered to be a force that acts as a supportive and promoting function from <the experience of the yips $>$ to <overcoming the yips $>$ and $<$ psychological growth as an athlete $>$. With this SG, it is thought that the factors and their roles in the experience of the yips and the psychological changes associated with overcoming the disorder became searchable. In addition, we have shown in Table 3 (based on Wada, 2016: p. 50) the meaning of the main concepts in the above TEM and positioning in the present study.

\subsection{Stages of the Path toward Psychological Growth in an Athlete Who Experienced the Yips and Overcame the Affliction}

The path from experiencing the yips to psychological growth as an athlete (accompanied by overcoming the yips) was considered to be composed roughly of two stages. That is, the period from <the experience of the yips $>$ to $<$ passive coping behavior > was set as "the period of psychological conflict and struggle,"

Table 1. Examples of headings and narratives concerning $<$ the experience of the yips $>$.

\begin{tabular}{|c|c|c|}
\hline & $\begin{array}{l}\text { Example } \\
\text { headings }\end{array}$ & Example narratives \\
\hline \multirow{3}{*}{$\begin{array}{l}\text { Experience } \\
\text { of the yips }\end{array}$} & $\begin{array}{l}\text { Cannot control } \\
\text { my body }\end{array}$ & $\begin{array}{l}\text { I thought, "Next, I'll do this," but my body wouldn't move as I } \\
\text { wanted... Stuff like that happened. }\end{array}$ \\
\hline & $\begin{array}{l}\text { Abnormal } \\
\text { sensations }\end{array}$ & $\begin{array}{l}\text { It's like I couldn't feel the ball in my hand... I knew that I } \\
\text { threw it, but I didn't know where. }\end{array}$ \\
\hline & $\begin{array}{l}\text { Cannot control } \\
\text { the ball when } \\
\text { throwing and } \\
\text { pitching }\end{array}$ & $\begin{array}{l}\text { Basically, all my throws were wild ones, so when a senior told } \\
\text { me "Just throw it low. It's fine if it bounces once or twice," I } \\
\text { really... I just threw it one or two meters, like a grounder. }\end{array}$ \\
\hline
\end{tabular}


Table 2. Examples of headings and narratives concerning < psychological growth as an athlete $>$.

\begin{tabular}{|c|c|c|}
\hline EFP & Example headings & Example narratives \\
\hline & \multirow[t]{2}{*}{$\begin{array}{l}\text { Change in attitude } \\
\text { and consciousness } \\
\text { toward baseball }\end{array}$} & $\begin{array}{l}\text { I was told that "playing catch is the basic" all through elementary, middle, and high school, like } \\
\text { "playing catch is the most important thing." I said "yes," but that was obvious... not about whether } \\
\text { it was basic... I thought it was obvious, so when I couldn't throw anymore, I realized again how } \\
\text { important playing catch is. You can't play baseball unless you do this [throwing] properly... I } \\
\text { thought it's not baseball otherwise... Since then (before uni), I'd goof around playing catch with } \\
\text { underarm throws... throwing curveballs... Now, I haven't thrown once... none of those... If the } \\
\text { other guy throws it to me... and says, like, "Try a curve ball," I'll say, "No, I won't throw." He'll be, } \\
\text { like, "That'll sure make it weird for you." }\end{array}$ \\
\hline & & $\begin{array}{l}\text { My way of thinking changed... Maybe I'll become better if I do more like this? I came to think } \\
\text { there's still so much I can do..., like, I became a bit more serious, a bit more earnest... about } \\
\text { baseball... not taking it lightly..., but I came to think that I should really put thought into it. }\end{array}$ \\
\hline & & $\begin{array}{l}\text { [Back when I had the yips symptoms] I disliked baseball. I disliked playing baseball... I didn't want } \\
\text { to go to practice and felt like I wanted to quit many times... I felt so, but ever since I became able to } \\
\text { throw again, I've been thinking maybe I can change more... Like, maybe I can improve... I was so } \\
\text { bad at throwing, but now I can throw... It's like I can improve if I work hard... I started thinking I } \\
\text { can improve. }\end{array}$ \\
\hline
\end{tabular}

Self-understanding I was always placing [in] games as vice-captain and a regular throughout elementary and middle school; I joined a strong team in high school and was in the lineup pretty often, so I thought, "I'm pretty awesome," although I didn't let it show... I went to a good high school, and we played at Koshien, stuff like that... If someone else couldn't play the way I wanted, I'd be, like, "This guy's hopeless; get your act together." I wouldn't say it out loud, but that's what I thought. But in the end, because this happened [the experience of the yips], I felt that I sucked too... like, because I

Psychological growth as an athlete EFP
Understanding and acceptance of others
Back in high school and middle school, if somebody kept throwing me wild ones while playing catch, I'd get irritated, like "Throw seriously." I wouldn't say it out loud, but I'd think it in my head... like, "Why can't you throw?" Things like that... Ever since I lost my ability to throw, I'd be throwing quite a lot of them [wild throws] to the one I was playing catch with... I'd say, "I'm really sorry," and he'd say, "Don't sweat it." Since then, I... before, if someone would throw me a wild one when playing catch, I'd think, "Throw it," but I never think like that now... because I threw so many wild ones before myself... Like, this is nothing compared to that... I came to think, "He's so much better than me." Like, I don't get irritated by wild throws... That changed... I think so at least. Like, I've become more forgiving... stuff I can't do... I'm able to think that way now even if someone throws me a wild one. Forgiving,... that's right... I stopped being short-tempered... Like, I don't get angry so much... I've stopped getting irritated.

I've received so much advice, and I've caused a lot of trouble by throwing wild balls and tosses.. We'd play catch before the fungoes, and the other person would be preparing for the fungoes, but I'd just throw wild throws, and we wouldn't do it properly before the fungoes... I'd feel sorry for him, but he wouldn't get upset despite my wild throws... I'd say, "Sorry," and he'd say, "It's fine." I came to feel like that.
Wanting to help others with experience of the yips
If a junior student, a person like that [someone suffering from the yips], came along- $\mathrm{X}$ [a senior student on Participant A's current team and an athlete who experienced the yips]. Matsuo would give quite a lot of advice too... I kind of want to do something like that, too... I used to think, "I'm number one" (before experiencing the yips), but I'm thinking that I'd like to help out my juniors if there's anyone who can't throw.

It's, like, I want to give some guidance... Like, if I see someone like that [an athlete suffering from the yips], I do want to give some advice... I came to think that I wanted to give some advice. 
Table 3. Principal TEM concepts' meaning and relevance to the present study.

\begin{tabular}{|c|c|c|}
\hline Concept & Meaning & Relevance to the present study \\
\hline Equifinality Point (EFP) & $\begin{array}{l}\text { A point at which } \\
\text { diverse paths merge }\end{array}$ & $\begin{array}{l}\text { <overcoming the yips }> \\
\text { <psychological growth as an athlete }>\end{array}$ \\
\hline Polarized EFP (P-EFP) & $\begin{array}{c}\text { A point of } \\
\text { complementary EFPs }\end{array}$ & $\begin{array}{c}<\text { not overcoming the yips }><\text { not perceiving } \\
\text { psychological growth as an athlete }>\end{array}$ \\
\hline $\begin{array}{l}\text { Obligatory Passage } \\
\text { Point (OPP) }\end{array}$ & $\begin{array}{l}\text { A point experienced by } \\
\text { most people }\end{array}$ & $\begin{array}{c}<\text { negative psychological change }> \\
\quad<\text { passive coping behavior }>\end{array}$ \\
\hline Social Guidance (SG) & $\begin{array}{l}\text { A force that supports } \\
\text { movement toward the EFP }\end{array}$ & [social support] \\
\hline
\end{tabular}

whereas the period from <intentional and positive meaning-making for the yips and throwing $>$ to $<$ psychological growth as an athlete $>$ was established as "the period of psychological growth as an athlete." Herein, we explain each period by citing a sample statement from Participant A.

\subsection{The Period of Psychological Conflict and Struggle}

Immediately after $<$ the experience of the yips $>$ (Table 1), Participant A passed through the OPP (<negative psychological changes $>$ ); as shown in Table 4, this stage involved "anxiety," "obsession," "excessive awareness," "loss of confidence," "concern about others' opinions," and "arousal of a desire to escape or withdraw." Sample statements from Table 4 include the following: "It's like I don't want to face baseball. I came to dislike the word "baseball,", and "I want to quit baseball; I don't want to practice." These responses indicate that $<$ the experience of the yips> had a major influence on Participant A's life as an athlete. To soften the psychological suffering that came with $<$ the experience of the yips $>$, Participant A said that he repeated a $<$ passive coping behavior $>$ as a temporary measure (Table 4). However, this <passive coping behavior $>$ could not immediately fix the condition and its symptoms.

The cycle of struggling associated with $<$ the experience of the yips $>$, <negative psychological changes $>$, and $<$ negative coping behavior $>$ is called the "period of psychological conflict and struggle."

\subsection{The Period before Psychological Growth as an Athlete}

After going through the period of psychological conflict and struggle, or as a way to break out of that period, Participant A performed <intentional and positive meaning-making for the yips and throwing $>$, as indicated by the following narrative:

The worst time was ... it was the worst around May.... I didn't want to go to practice throughout that period.... It was like that throughout May and June, but from around the time the summer break started in July, from when I started trying to think positively..., it became totally different. 
Table 4. Example of headings and narratives concerning < arousal of negatives effect $><$ passive coping behavior $>$.

\begin{tabular}{|c|c|c|}
\hline OPP & $\begin{array}{l}\text { Example } \\
\text { headings }\end{array}$ & Example narratives \\
\hline \multirow{6}{*}{$\begin{array}{l}\text { Negative } \\
\text { psychological } \\
\text { change }\end{array}$} & Anxiety & $\begin{array}{l}\text { Maybe when I'd be in the room by myself and turn the lights off, thinking, "OK, let's sleep," like, "maybe } \\
\text { we'll do fungoes at practice tomorrow." }\end{array}$ \\
\hline & Obsession & $\begin{array}{l}\text { In the past, I really couldn't throw... especially not to seniors... and third-years... There will be } \\
\text { punishment when doing relays... when playing catch relays and passing the ball on... I thought, } \\
\text { "I have to throw it properly." And I was relatively comfortable throwing to guys in my own year... } \\
\text { compared to third-years... Then I'd be able to throw without thinking too much. }\end{array}$ \\
\hline & $\begin{array}{l}\text { Excessive } \\
\text { awareness }\end{array}$ & $\begin{array}{l}\text { Also while waiting in the defense, I'd be thinking about the next ball. If I'd fail with the first ball, I'd } \\
\text { immediately think, like, "Next one, I'll do like this and this." I kept thinking like that. }\end{array}$ \\
\hline & $\begin{array}{l}\text { Loss of } \\
\text { confidence }\end{array}$ & $\begin{array}{l}\text { After that, I started thinking, like, "Maybe my way of throwing is bad." I started to think like that after } \\
\text { that. }\end{array}$ \\
\hline & $\begin{array}{l}\text { Concern about } \\
\text { others' } \\
\text { opinions } \\
\text { of you }\end{array}$ & $\begin{array}{l}\text { As expected, when I first joined-I'm from XX Prefecture (birthplace)_, but... there were so few... from } \\
\text { the same... I wondered how I should interact with them... I soon lost my ability to throw, so in the } \\
\text { beginning... someone in my year asked, "You know how to throw?" So I didn't talk so much about it in } \\
\text { the beginning, right? And I'd be, like, "Yeah." Kind of embarrassed... I thought maybe they made fun of } \\
\text { me behind my back... I'd be thinking like that... Seniors would be laughing too, and I'd be } \\
\text { thinking, "They're all making fun of me." }\end{array}$ \\
\hline & $\begin{array}{l}\text { Tendency to } \\
\text { escape or } \\
\text { withdraw }\end{array}$ & $\begin{array}{l}\text { When I lost my ability to throw,... I said this before too, but I did want to quit baseball... I didn't want to } \\
\text { practice... Like, "What's the point of baseball?" I even thought like that. }\end{array}$ \\
\hline \multirow{4}{*}{$\begin{array}{l}\text { Passive coping } \\
\text { behavior }\end{array}$} & $\begin{array}{l}\text { Inappropriate } \\
\text { play }\end{array}$ & $\begin{array}{l}\text { Also during soft toss batting,... I couldn't even throw in arches... It slipped, so I'd sometimes hit the } \\
\text { batter to the right. }\end{array}$ \\
\hline & $\begin{array}{l}\text { Avoiding or } \\
\text { escaping from } \\
\text { throwing }\end{array}$ & $\begin{array}{l}\text { Like, I'll keep it secret that I can't throw... I'd commit an error on purpose and not throw... That's } \\
\text { right... Get an error... On to the next... Get an error... Pretend to throw and on to the next... and then, } \\
\text { just pass the ball to the next person. }\end{array}$ \\
\hline & $\begin{array}{l}\text { Excessively } \\
\text { looking up } \\
\text { coping } \\
\text { methods }\end{array}$ & $\begin{array}{l}\text { I started thinking about it quite a lot and would look up ways to fix it on my phone and look around the } \\
\text { Web... Well, I watched videos liked that online and on Youtube as well. }\end{array}$ \\
\hline & $\begin{array}{l}\text { Changing the } \\
\text { person you } \\
\text { throw to }\end{array}$ & $\begin{array}{l}\text { When playing catch, even before practice started, I'd say to a first-year, "Let's play catch today," because } \\
\text { if we were uneven (the number of people practicing), then I'd maybe have to play with a senior. }\end{array}$ \\
\hline
\end{tabular}

In the TEM graph presented in this study, this point is seen to be the turning point toward two EFPs, <overcoming the yips $>$ and $<$ psychological growth as an athlete $>$. Then, following $<$ intentional and positive meaning-making for the yips and throwing $>$, Participant A said that he performed <positive technical coping behavior $>$, meaning an active trial and error approach toward his technique in an effort to achieve <support seeking $>$. In other words, he was actively seeking support in his efforts for <overcoming the yips>:

I guess ... I changed my way of thinking. I was ready to just throw wild pitches.... Like, wild throws would be the norm.... I was thinking it'd be a feat if I could throw it (where I aimed)...There were some freshmen, first 
and second basemen.... I, like, told them that I'd be throwing wild ones today, so "catch them if you can." They'd laugh and say stuff like "Just throw it." I'd also tell my seniors, "I'll be throwing wild ones today, so please back me up" or something like that. (support seeking $>$ )

I stopped watching stuff like that online or on YouTube ... to stop myself from overthinking.... My seniors would also give me quite a lot of advice ... things like, "You're just throwing with your upper body" and "Your chest is closed." "Just do a bit more like this." I'd try those things out next time I played catch on my own time.... And once I'd become comfortable with it, I'd want to incorporate it when playing catch the next day.... That's how it turned out. (<positive technical coping behavior $>$ )

From this narrative, we found that Participant A-by having performed $<$ intentional and positive meaning-making for the yips and throwing $>$ - sought support from those surrounding him through repeated trial and error.

As Participant A had passed from <intentional and positive meaning-making for the yips and throwing $>$ to < support seeking $>$ or $<$ positive technical coping behavior $>$, his symptoms and psychological issues began to produce remarkable positive changes. Further, he was able to achieve an EFP (i.e., <overcoming the yips $>$ ) with positive and continuous efforts. Participant A talked about the process for reaching this EFP as follows:

Like, wild throws would be the norm...; I came to think that actually throwing a good ball would be amazing instead.... My ball play completely changed when I started thinking like that.... My fingers would steer the ball and I'd throw good balls.... In the beginning, I did [have] wild throws, but cared so much about what others thought that I thought I had to throw a good one next... Now, I'm able to throw normally.... This made it fun to throw balls.... I'm still throwing normally with all my strength.

As indicated above, changing his way of thinking about the yips or throwing balls was the turning point for <overcoming the yips>, which imbued Participant A with <psychological respite $>$. Reflecting on his past experiences with composure facilitated his $<$ psychological growth as an athlete $>$, which consists of the following: "the change in attitude and consciousness toward baseball," self-understanding, an understanding of others, and acceptance of people who have experienced the yips (see Table 4).

\subsection{Sociocultural Influences Ranging from the Experience of the Yips to Overcoming the Affliction or Psychological Growth as an Athlete}

In the process leading from $<$ the experience of the yips $>$ to $<$ overcoming the yips $>$, and in the process from $<$ the experience of the yips $>$ to $<$ psychological growth as an athlete>, [social support] has a promoting and supporting function, as seen in the following narrative:

My uni classmates would say stuff like... "Just keep going a bit more," 
"You're already better so it's just a bit more." Stuff like that kept me from quitting baseball, I think.

If I threw a good ball, they'd say something like "You were good today" or

"You played great today." I think things started changing from there.

As suggested by the above narrative, Participant A's active and continuous coping behavior with regard to the yips might have been encouraged by dedicated [social support] from the people surrounding him. Moreover, the narrative below indicates that the dedication [social support] from athletes who have experienced the yips contributed to the alleviation of psychological suffering and <overcoming the yips>:

Even when I couldn't throw, there were those around me who'd say stuff like, "I can't throw either. Don't sweat it" or "You'll get there." That was another reason I got better...; I felt more at ease somehow.

At the same time, the narrative below (excerpt from Table 2) also suggests that [social support] had a promoting and supporting function in the process of $<$ psychological growth as an athlete $>$ as well:

If a junior student, a person like that (someone suffering from the yips), came along, ... X (a senior student on Participant A's current team and an athlete who has experienced the yips) would give quite a lot of advice too.... I kind of want to do something like that too.... I used to think, "I'm number one (before experiencing the yips)," but I'm thinking that I'd like to help out my juniors if there's anyone who can't throw.

There are many similar narratives besides the one above, that clearly show that [social support] had a promoting and supporting function in Participant A's progress from $<$ the experience of the yips $>$ to overcoming the yips $>$ and $<$ psychological growth as an athlete $>$. Furthermore, it was also indicated that receiving dedicated [social support] could cause a positive change in a person's relationship with those around them, as shown in the narrative below:

I had classmates telling me that I'd improved. More and more conversations like that.... It's like it made it easier to throw.... They'd say stuff like, "When I'm catcher, it's fine if you throw me a wild pitch" or "I'll cover for you if you throw a low ball." That made me talk more.... Or it's more, like, in order to communicate well ... sometimes communication improves after you stop being able to throw.

\section{Discussion}

The present study involved research and analyses to investigate the process beginning with the experience of the yips to psychological growth as an athlete. As a result, the process of psychological growth as an athlete associated with Participant A's experience of the yips was divided into the period of psychological conflict and struggle and the period leading to psychological growth as an ath- 
lete. In addition, from the narrative of Participant $\mathrm{A}$, we considered the period and factors that seemed to play important roles in the aforementioned process.

\subsection{Significance of the Period of Psychological Conflict and Struggle in the Process of Psychological Growth as an Athlete Accompanying the Experience of the Yips}

Participant A followed the path of $<$ negative psychological changes $>$ and $<$ passive coping behavior $>$, along with $<$ the experience of the yips $>$. In the present study, these two passing points were determined as the OPP, for the reasons described below. First, in a study focusing on the psychological aspect of the athletes who have experienced the yips, similar reports can be found (e.g., Mukai, 2016) That is, concerning negative psychological changes accompanying the experience of the yips, for example, the occurrence of negative emotions such as anxiety, fear, anger, loss of confidence, and concern about mistakes have been reported (Bawden \& Maynard, 2001; Philippen \& Lobinger, 2012).

It has also been revealed that athletes who have experienced the yips repeat negative coping behaviors when negative psychological changes that accompany the experience of the yips occur (Iwata \& Hasegawa, 1981; Nishino et al., 2006). For instance, Nakagomi (2004) reported that athletes who have experienced the yips and who have engaged in mental training might think, "I mustn't hit the batter" or "I have to let him hit it" during batting practice, which leads them to throw easy balls. That is, they change their play style temporarily because they are afraid that the symptoms of the yips could surface.

Furthermore, even in studies focusing on a serious negative experience in a daily living situation, it has been pointed out that "when people personally experience an event that is difficult to cope with, they come to feel emotions like suffering, anger, and anguish about that event" (Hatori \& Kodama, 2012: p. 12). It has also been reported that they try to gloss over such negative emotions, stop any factors that stimulate those emotions, or run away from them (Greenberg \& Pavio, 1997). Bearing in mind these insights, it is difficult to imagine anyone with an experience of the yips who has no negative emotions or thoughts whatsoever or who does nothing to cope with a situation when they have such emotions or thoughts and cannot play as usual. Based on the above, we may surmise that the two factors of <negative psychological changes $>$ and <passive coping behavior $>$ are "checkpoints that most people normally have to pass through" (Kawai et al., 2016: p. 4)-hence, establishment of the OPP.

The Handbook of Posttraumatic Growth (Janoff-Bulman, 2006: p. 87) cites expressions like "What doesn't kill us makes us stronger" and "No pain, no gain" from philosophical and religious texts to show that people grow by facing psychological conflict and struggle or as part of the process of doing so.

It appeared that Participant A was thinking it natural that throwing could be done at will. However, for Participant A, the experience of the yips meant that throwing and pitching at will, which had previously been a given, suddenly was no longer so obvious. It is thought that this was a shocking realization for him. 
Moreover, with the symptoms of the yips appearing for as long as four months, Participant A had a long-term struggle. Experiences such as this can become an opportunity for gaining a new perspective or thinking about pitching, throwing, or other baseball skills (e.g., batting, running, and catching), and it has been suggested that such reassessment can bring about psychological growth in the form of a changed attitude and awareness toward baseball, for example.

Moreover, taking the above into consideration, we may surmise that $<$ negative psychological changes $>$ and <negative coping behavior $>$ could be the OPP that most people experiencing the yips have to pass through (because psychological growth will necessarily accompany the experience of the yips). That is, it has been suggested that the cycle of conflict and struggle that is $<$ the experience of the yips>, <negative psychological changes>, and <passive coping behavior $>$ could play a major role in the process of psychological growth that accompanies the experience of the yips.

\subsection{Significance of Intentional and Positive Meaning-Making for the Yips and Throwing in the Process of Psychological Growth as an Athlete that Accompanies the Experience of the Yips}

One day, Participant A suddenly could no longer throw as he wanted to; he was repeatedly throwing obviously wild pitches. This experience caused him many <negative psychological changes> and exposed him to a crisis as a baseball player. Horita and Sugie (2012: p. 113) pointed out, "When exposed to events that threaten one's existence, meaning stressful and critical situations, people are motivated to perform new meaning-making in order to restore a sense of meaning to their coping and life that follows." Thus, we may surmise that Participant A experienced the yips and was exposed to a crisis as a baseball player, which led him to perform new meaning-making with regard to his previous perspective on throwing and pitching.

Additionally, it has been pointed out that the repetition of temporary coping behaviors, such as throwing to a different person, changing form, and practicing avoidance, tend to represent $<$ negative coping behavior $>$ and may lead to unfavorable outcomes (e.g., Suls et al., 1996). For instance, Billings and Moos (1984) reported that avoidance for coping with stress is positively correlated with depression. Further, it is possible that excessively looking up coping methods, which tends to be a <negative coping behavior $>$, causes "negative rumination," which is defined as "the tendency to continue to think about something bad, harmful, or unhopeful for a long time" (Ito et al., 2006: p. 763). In other words, excessively searching online or in books for ways to cope with the yips could lead to overthinking regarding negative events of one's experience of the yips. Many studies have suggested that such negative rumination on negative life experiences can cause symptoms of depression and stress (e.g., Nolen-Hoeksema \& Morrow, 1991; Treynor et al., 2003). Considering previous studies that are similar, we suspect that the long-term repetition of <negative coping behavior $>$ following <negative psychological changes $>$ associated with $<$ the experience of the 
yips $>$ can lead to further <negative psychological changes $>$. Furthermore, if the symptoms of depression and stress lead to more serious conditions, there is a risk that the afflicted person may quit playing his/her sport.

However, by performing <intentional and active meaning-making with regard to the yips $>$, Participant A was able to leave the period of psychological conflict and struggle. Moreover, he was able to direct himself toward the EFP in the form of <overcoming the yips $>$ and $<$ psychological growth as an athlete $>$. That is, it is possible that <intentional and active meaning-making with regard to the yips $>$ plays an extremely important role in the process of psychological growth as an athlete following the experience of the yips by bringing about a major turning point in one's life.

Previous studies have also pointed out the importance of "meaning-making processes" (Horita \& Sugie, 2013: p. 409) in the development of psychological growth that accompanies negative experiences, which demonstrates how the individual copes with negative experiences cognitively and emotionally (e.g., Barrington \& Shakespeare, 2013). Representative examples of concepts that explain this process of meaning-making are the concepts of assimilation and accommodation proposed by Joseph and Linley (2005). Assimilation is defined as "interpreting and comprehending the experience so that it conforms with one's perspective and [way of] thinking about things" (Horita \& Sugie, 2013: p. 410), while accommodation is defined as "consciously and intentionally taking cognitive and emotional measures to understand and interpret that experience, thereby adjusting one's perspective and [way of] thinking about things" (Horita \& Sugie, 2013: p. 410). Of these two concepts, it has been pointed out that meaning-making through accommodation correlates with psychological change associated with negative experiences (Davies et al., 2000; Joseph \& Linely, 2006). In other words, rather than cognitively and emotionally coping with an event without changing one's perspective and way of thinking about things, psychological growth is better promoted through meaning-making through accommodation wherein one intentionally and actively adjusts his or her perspective or way of thinking about things. Since we can find features that resemble meaning-making through accommodation-which is said to have a stimulating effect on psychological growth associated with negative experiences-, in the narrative of Participant A, the findings of the present study can be said to support some of the conclusions from previous studies.

If we consider the above, when athletes who have experienced the yips demonstrate some kind of psychological growth associated with the experience of the yips, <intentional and active meaning-making with regard to the yips> plays an extremely important role and is possibly an indispensable coping behavior.

\subsection{The Role of Social Support in the Process of Psychological Growth as an Athlete Who Has Experienced the Yips and Overcome the Affliction}

It has been suggested that dedicated [social support] was an important part of 
the process leading Participant A from his experience of the yips to the EFP of $<$ overcoming the yips $>$ and $<$ psychological growth as an athlete $>$.

First, let us examine the potential promoting function of social support for overcoming the yips. It has been suggested that because Participant A kept receiving dedicated [social support], he was stimulated to think, "I want to fix it somehow" and "I can't lose to this." Such thoughts allowed him to perform active and continuous coping. Many studies have already proven the importance of dedicated social support in coping with negative events in sports (Johnston \& Carroll, 1998; Podlog et al., 2011; Rees et al., 2010). For instance, Suzuki and Nakagomi (2013) pointed out the importance of social support by identifying its effects in the rehabilitation of injured athletes as "increasing concentration on the rehabilitation," "increasing the desire to make a quick comeback," and "sustaining and amplifying the motivation to compete," which are features that resemble the effects of [social support] in Participant A's process of coping with the yips. Bianco (2001) identified relieving stress and sustaining motivation for rehabilitation as effects of dedicated social support on injured athletes. These previous studies also give us ample reason to believe that such support from people surrounding the individual may have an important function in overcoming the yips.

Next, let us examine the potential promoting function of social support from the experience of the yips to psychological growth as an athlete.

Many studies have demonstrated that an individual who has experienced negative events can be buoyed toward psychological growth by dedicated social support from the people surrounding him/her during the process of coping with those events (Cryder et al., 2006; Tsai et al., 2015). For instance, Taku (2016) pointed out that there are different types of psychological growth that accompany negative experiences; of these, psychological growth associated with relationships with others (e.g., stronger compassion for the other person, an understanding of the value of family and friends) is brought about by dedicated support from surrounding people. Moreover, Hiraki (2003) and Iimura (2016) provided narratives from victims of volcano eruptions, earthquakes, and other disasters whose receipt of substantial support led them to want to help others in similar situations.

Similar reports have been made in the field of sports psychology (Fletcher \& Sarkar, 2012). For instance, Galli and Reel (2012) pointed out that dedicated social support for those coping with negative events in their sport has been proven to promote psychological growth in persons facing such events. Examples of psychological growth also include heightened consciousness about the importance of support from others and a stronger will to support others in similar situations. By the same token, Galli and Vealey (2008) identified "gained realization of support" and "gained motivation to help others" as psychological growth associated with experiences of adversity in sports; they mentioned that dedicated social support plays an important role in psychological growth. These findings 
suggest that psychological growth, such as <understanding and accepting others $>$ and <increased motivation to support athletes who have experienced the yips $>$ can be facilitated by dedicated social support from surrounding people, as also shown in the narrative examples in Table 2.

Moreover, it has been reported that dedicated social support for athletes who have faced negative events can encourage their adaptive coping with and positive meaning-making of those events (Galli \& Reel, 2012). Therefore, we cannot deny the possibility that dedicated social support for athletes who have experienced the yips does not only directly contribute to overcoming the yips and exhibiting psychological growth as an athlete; it also has indirect effects. For instance, it could change the athlete's meaning-making of the experience of the yips into something positive.

Incidentally, these effects of social support can be explained with the concept of contingency, proposed by Yasuda et al. (2008). "With time, experiences are thought to undergo new qualitative changes through encounters and interactions with events and other people," and contingency signifies "the process that brings about change" (Sato, 2009: p. 68). Furthermore, contingency as discussed here does not refer only to random events; it also means "a singular, inevitable, and unrepeatable phenomenon, something that contains new possibilities and brings about qualitative change to later experiences because of encounters and interactions with certain events and people" (Sato, 2009: p. 68). We surmise that the dedicated [social support] during Participant A's process of coping with the yips brought about not only qualitative change in the form of negative psychological changes, but also new change in the form of psychological growth as an athlete.

When considering what has been reported in the aforementioned studies, findings from Participant A's narratives and the TEM graph suggest that psychological growth as an athlete associated with the experience of the yips is not merely fostered within the affected individual, but it is psychological growth promoted by trainers, past and current teammates, and family members surrounding the athlete. Furthermore, the athlete's diverse interactions with his/her surroundings play a role in this growth.

\subsection{The Meaning of Overcoming the Yips in the Process of Obtaining Psychological Growth as an Athlete Following the Experience of the Yips}

Participant A experienced the yips and underwent psychological turmoil and conflict, but he earnestly faced his circumstances and attempted to overcome the affliction through trial and error. His efforts were rewarded, as he overcame the yips. Is it not possible that this series of experiences led Participant $A$ to develop confidence and discover new possibilities as a baseball player? Narratives that indicate positive changes associated with overcoming the yips are provided in Table 2.

This process of psychological growth brought about by coping with and over- 
coming negative events, has also been reported in previous studies about PTG. For instance, individuals who have experienced loss, natural disasters, and disease seem to have gained confidence by overcoming those difficult experiences (Taku, 2016). Furthermore, they tend to recognize their own strength (Calhoun \& Tedeschi, 2006). Similar observations have been made with regard to psychological growth associated with negative experiences in sports. For instance, Galli and Vealey (2008: p. 330) pointed out that "with few exceptions, the athletes expressed having successfully overcome adversity and in most cases achieving personal growth because of it." This suggests that overcoming the yips contributes to psychological growth as an athlete.

By the same token, overcoming the yips can be seen as something that allows athletes who have experienced the yips to realize their personal growth in terms of their sport. We can imagine that some athletes who have experienced the yips are unable to or lack the composure to notice positive changes as long as symptoms of the yips are apparent, simply because of negative emotions and thoughts or because they continue to focus on overcoming the yips. As such, we cannot deny the possibility that overcoming the yips may provide athletes who have experienced the disorder with a first step toward psychological respite for noticing their growth.

The above leads us to surmise that overcoming the yips potentially promotes psychological growth as an athlete, and it can serve as a first step for the athlete to notice the psychological growth that accompanies the experience of the yips.

\section{Conclusion}

In the present study, the process of psychological growth as an athlete associated with the experience of the yips was examined through the narrative of a university baseball player who experienced and overcame the disorder (Participant A). The findings have been presented in a TEM. It is suggested that psychological conflict and struggle following the experience of the yips, cognitive coping to intentionally change his thinking about the yips and throwing (intentional and active meaning-making with regard to the yips and throwing), dedicated social support from the people surrounding him, and overcoming the yips were intimately connected with Participant A's consequent psychological growth as an athlete. Above all, we surmise that intentional and positive meaning-making for the yips and throwing served as a turning point that led from the process of psychological conflict and struggle following the experience of the yips to overcoming it and developing psychological growth as an athlete. These findings support the research findings of previous studies dealing with psychological growth associated with negative experiences in the fields of general, positive, and sports psychology.

Meanwhile, we acknowledge several limitations of our paper as follows. The present study dealt with a single case and was not meant to be representative of the process of psychological growth among athletes following their experiences 
of the yips and overcoming the disorder. Prior researchers have pointed out that single case studies provide poor evidence for practice, but they can generate hypotheses for later studies (e.g., Daly et al., 2007). The present study suggests that psychological conflict and struggle following the experience of the yips, intentional and active meaning-making with regard to the yips and throwing, and social support played important roles in Participant A's psychological growth as he overcame the affliction.

\section{Conflicts of Interest}

The authors declare no conflicts of interest regarding the publication of this paper.

\section{References}

Adler, C. H. (2012). Sports-Related Task-Specific Dystonia: The Yips. In M. Stacy (Ed.), Neurology Handbook of Dystonia (2nd ed., pp. 209-217). London: Informa Healthcare. https://doi.org/10.3109/9781420019988.014

Affleck, G., Tennen, H., \& Gershman, K. (1985). Cognitive Adaptations to High-Risk Infants: The Search for Mastery, Meaning, and Protection from Future Harm. American Journal of Mental Deficiency, 89, 653-656.

Aoki, K., \& Matsudamoto, K. (1999). Psychological Risk Factors and Athletic Injury. Bulletin of the School of Nursing, Yamaguchi Prefectural University, 3, 9-19.

Barrington, A. J., \& Shakespeare-Finch, J. (2013). Working with Refugee Survivors of Torture and Trauma: An Opportunity for Vicarious Post-Traumatic Growth. Counselling Psychology Quarterly, 26, 89-105. https://doi.org/10.1080/09515070.2012.727553

Bawden, M., \& Maynard, I. (2001). Towards an Understanding of the Personal Experience of the "Yips" in Cricketers. Journal of Sports Sciences, 19, 937-953. https://doi.org/10.1080/026404101317108444

Bennett, J., Hays, K., Lindsay, P., Olusoga, P., \& Maynard, I. W. (2015). Yips and Lost Move Syndrome: Exploring Psychological Symptoms, Similarities, and Implications for Treatment. International Journal of Sport Psychology, 46, 61-82.

Bianco, T. (2001). Social Support and Recovery from Sport Injury: Elite Skiers Share Their Experiences. Research Quarterly for Exercise and Sport, 72, 376-388. https://doi.org/10.1080/02701367.2001.10608974

Billings, A. G., \& Moos, R. H. (1984). Coping, Stress, and Social Resources among Adults with Unipolar Depression. Journal of Personality and Social Psychology, 46, 877-891. https://doi.org/10.1037/0022-3514.46.4.877

Calhoun, L. G., \& Tedeschi, R. G. (2006). The Foundations of Posttraumatic Growth: An Expanded Framework. In L. G. Calhoun, \& R. G. Tedeschi (Eds.), Handbook of Posttraumatic Growth, Research and Practice (pp. 3-23). Mahwah, NJ: Lawrence Erlbaum Associates.

Clarke, P., Sheffield, D., \& Akehurst, S. (2015). The Yips in Sport: A Systematic Review. International Review of Sport and Exercise Psychology, 8, 156-184. https://doi.org/10.1080/1750984X.2015.1052088

Cryder, C. H., Kilmer, R. P., Tedeschi, R. G., \& Calhoun, L. G. (2006). An Exploratory Study of Posttraumatic Growth in Children Following a Natural Disaster. American Journal of Orthopsychiatry, 76, 65-69. https://doi.org/10.1037/0002-9432.76.1.65

Daly, J., Willis, K., Small, R., Green, J., Welch, N., Kealy, M., \& Hughes, E. (2007). A Hie- 
rarchy of Evidence for Assessing Qualitative Health Research. Journal of Clinical Epidemiology, 60, 43-49. https://doi.org/10.1016/j.jclinepi.2006.03.014

Davis, C., Wortman, C.B., Lehman, D. R., \& Silver, R. (2000). Searching for Meaning in Loss: Are Clinical Assumptions Correct? Death Studies, 24, 497-540. https://doi.org/10.1080/07481180050121471

Dhungana, S., \& Jankovic, J. (2013). Yips and Other Movement Disorders in Golfers. Movement Disorders, 28, 576-581. https://doi.org/10.1002/mds.25442

Fletcher, D., \& Sarkar, M. (2012). A Grounded Theory of Psychological Resilience in Olympic Champions. Psychology of Sport and Exercise, 13, 669-678. https://doi.org/10.1016/j.psychsport.2012.04.007

Flick, U. (2007). Qualitative Forschung-Ein Handbuch [An Introduction to Qualitative Research: Methodology for Human Science] (3rd ed.). Reinbek: Rowohlt Verlag $\mathrm{GmbH}$.

Galli, N., \& Reel, J. J. (2012). “It Was Hard, But It Was Good”: A Qualitative Exploration of Stress-Related Growth in Division I Intercollegiate Athletes. Qualitative Research in Sport, Exercise and Health, 4, 297-319. https://doi.org/10.1080/2159676X.2012.693524

Galli, N., \& Vealey, R. S. (2008). “Bouncing Back” from Adversity: Athletes’ Experiences of Resilience. The Sport Psychologist, 22, 316-335. https://doi.org/10.1123/tsp.22.3.316

Glaser, B. G., \& Strauss, A. L. (1967). The Discovery of Grounded Theory: Strategies for Qualitative Theory. New Brunswick: Aldine Transaction.

Greenberg, L. S., \& Pavio, S. C. (1997). Working with Emotions in Psychotherapy. New York: The Guilford Press.

Hanashima, H. (2018). Experiences of Parents with a Socially Withdrawn Son: From Transition to Social Withdrawal and Overcoming Critical Situations. Japanese Journal of Qualitative Psychology, 17, 25-42.

Harada, H., Konishi, M., Teraoka, S., \& Ura, M. (2009). Development of Public Health Nurse's Relational Skills: Focusing on Homecare Services for the Mentally Challenged. The Japanese Journal of Experimental Social Psychology, 49, 72-83. https://doi.org/10.2130/jjesp.49.72

Hatori, K., \& Kodama, M. (2012). Development of the Positive Acceptance of Adversity Scale. The Japanese Journal of Health Psychology, 25, 11-19. https://doi.org/10.11560/jahp.25.1_11

Hayashi, K., \& Tuchiya, H. (2012). Examination of Psychological Support Required for Olympians Considered from the Narrative of Their Own Experiences: Focusing on the Psychological Processes by Events and the Influences of Society. Japanese Journal of Sport Psychology, 39, 1-14. https://doi.org/10.4146/jjspopsy.2011-083

Hiraki, K. (2003). What Unzen Volcanic Survivors Get from the Adversity since the Eruption in 1990. Bulletin of Faculty of Contemporary Social Studies, Nagasaki Wesleyan University, 1, 21-29.

Horita, R., \& Sugie, M. (2012). Research on Meaning-Making Following Stressful Life Experiences: A Review. Tsukuba Psychological Research, 44, 113-122.

Horita, R., \& Sugie, M. (2013). The Influence of Meaning Making Following Stressful Life Experiences on Change of Self-Concept. The Japanese Journal of Psychology, 84, 408-418. https://doi.org/10.4992/jjpsy.84.408

Iimura, S. (2016). Perceptions toward Posttraumatic Growth: Life Crises and Positive Psychological Changes. Stress Management Research, 12, 54-65.

Ito, T., Takenaka, K., Tomita, T., \& Agari, I. (2006). Comparison of Ruminative Responses with Negative Rumination as a Vulnerability Factor for Depression. Psycholog- 
ical Reports, 99, 763-772. https://doi.org/10.2466/PR0.99.3.763-772

Iwata, I., \& Hasegawa, K. (1981). An Approach from the Standpoint of Sports Clinical Psychology to Psychogenic Motor Disorder. Japanese Society of Sport Psychology, 8, 28-34.

Janoff-Bulman, R. (2006). Schema-Change Perspectives on Posttraumatic Growth. In L. G. Calhoun, \& R. G. Tedeschi (Eds.), Handbook of Posttraumatic Growth, Research and Practice (pp. 81-99). Mahwah, NJ: Lawrence Erlbaum Associates.

Jansen, L., Hoffmeister, M., Chang-Claude, J., Brenner, H., \& Arndt, V. (2011). Benefit Finding and Post-Traumatic Growth in Long-Term Colorectal Cancer Survivors: Prevalence, Determinants, and Associations with Quality of Life. British Journal of Cancer, 105, 1158-1165. https://doi.org/10.1038/bjc.2011.335

Johnston, L. H., \& Carroll, D. (1998). The Provision of Social Support to Injured Athletes: A Qualitative Analysis. Journal of Sport Rehabilitation, 7, 267-284. https://doi.org/10.1123/jsr.7.4.267

Joseph, S., \& Linley, P. A. (2005). Positive Adjustment to Threatening Events: An Organismic Valuing Theory of Growth through Adversity. Review of General Psychology, 9, 262-280. https://doi.org/10.1037/1089-2680.9.3.262

Joseph, S., \& Linley, P. A. (2006). Growth Following Adversity: Theoretical Perspectives and Implications for Clinical Practice. Clinical Psychology Review, 26, 1041-1053. https://doi.org/10.1016/j.cpr.2005.12.006

Kagawa, M. (2013). Effect of Mental Training with Self-Monitoring and Cognitive Restructuring by Video Feedback for a College Baseball Player with Yips Symptom. Mental Training Journal, 7, 35-44.

Kagawa, M., \& Fukae, M. (2013). A Study on Psychological Trait of Junior High School Baseball Player with Symptom of Throwing and Catching Ataxia. Research Bulletin of Naruto University of Education, 28, 440-453.

Kasada, M. (2013). Life Course Choice Process of Mentally-Disabled Person's Brother: The Factor Which Led to a Solution of Discord and Maintenance for a Middle-Scense Brother. The Japanese Journal of Developmental Psychology, 24, 229-237.

Kawai, N., Kubota, Y., \& Kohno, S. (2016). Alumni's Social Adaptation Processes after Attending High School in Children's Self-Reliance Support Facilities: Analysis of the Trajectory Equifinality Model (TEM). The Japanese Journal of Criminal Psychology, $54,1-12$.

Le Floch, A., Vidailhet, M., Flamand-Rouvière, C., Grabli, D., Mayer, J. M., Gonce, M., Roze, E. et al. (2010). Table Tennis Dystonia. Movement Disorders, 25, 394-397. https://doi.org/10.1002/mds.22968

Linley, P., \& Joseph, S. (2004). Positive Change Following Trauma and Adversity: A Review. Journal of Traumatic Stress, 17, 1-21. https://doi.org/10.1023/B:JOTS.0000014671.27856.7e

Lyra, M. C., Valério, T. A. D. M., \& Wagoner, B. (2018). Pathways to Life Course Changes: Introducing the Concept of Avenues of Directive Meaning. Culture \& Psychology, 24, 443-459. https://doi.org/10.1177/1354067X18779060

Marquardt, C. (2009). The Vicious Circle Involved in the Development of the Yips. International Journal of Sports Science \& Coaching, 4, 67-88. https://doi.org/10.1260/174795409789577506

Matsuda, K., Susaki, Y., \& Sugiyama, Y. (2018). Relations between the Experience of the Yips and Athletes' Psychological Growth. SOJ Psychology, 5, 1-10. https://doi.org/10.15226/2374-6874/5/1/00144 
McDaniel, K. D., Cummings, J. L., \& Shain, S. (1989). The "Yips" a Focal Dystonia of Golfers. Neurology, 39, 192. https://doi.org/10.1212/WNL.39.2.192

Mukai, K. (2016). Study of the Psychological Process during the Yips in Baseball Experience Using the Trajectory Equifinality Model. Japanese Journal of Qualitative Psychology, 15, 159-170.

Mukai, K., \& Koga, S. (2017). Study of the Associations between Attention Style and Emergence/Persistence of Feeling Motion Difficulty Faced by Baseball Players. The Journal of Rehabilitation Psychology, 43, 73-84.

Nakagomi, S. (1987). A Psychotherapeutic Look at a Baseball Pitcher's Difficulty in Throwing the Ball at a Certain Distance: The Psychological Distance of Interpersonal Relationships Projected into the Distance of Throwing. Japanese Society of Sport Psychology, 14, 58-62.

Nakagomi, S. (2004). Clinical Psychology of Athlete. Tokyo: Dowashoin.

Nishino, S., Yamamoto, K., \& Oda, N. (2006). A Consideration on Psychogenic Disorder in Throwing Motion (Throwing Yips). Fukuoka University Review of Sports and Health Science, 18, 20-21.

Nolen-Hoeksema, S., \& Morrow, J. (1991). A Prospective Study of Depression and Posttraumatic Stress Symptoms after a Natural Disaster: The 1989 Loma Prieta Earthquake. Journal of Personality and Social Psychology, 61, 115-121. https://doi.org/10.1037/0022-3514.61.1.115

Ohkubo, T. (2001). Life Story Analysis: An Introduction to Qualitative Research. Tokyo: Gakubunsha.

Park, C. L., Cohen, L. H., \& Murch, R. L. (1996). Assessment and Prediction of Stress-Related Growth. Journal of Personality, 64, 71-105. https://doi.org/10.1111/j.1467-6494.1996.tb00815.x

Philippen, P. B., \& Lobinger, B. H. (2012). Understanding the Yips in Golf: Thoughts, Feelings, and Focus of Attention in Yips-Affected Golfers. The Sport Psychologist, 26, 325-340. https://doi.org/10.1123/tsp.26.3.325

Podlog, L., Dimmock, J., \& Miller, J. (2011). A Review of Return to Sport Concerns Following Injury Rehabilitation: Practitioner Strategies for Enhancing Recovery Outcomes. Physical Therapy in Sport, 12, 36-42. https://doi.org/10.1016/j.ptsp.2010.07.005

Rees, T., Mitchell, I., Evans, L., \& Hardy, L. (2010). Stressors, Social Support and Psychological Responses to Sport Injury in High- and Low-Performance Standard Participants. Psychology of Sport and Exercise, 11, 505-512. https://doi.org/10.1016/j.psychsport.2010.07.002

Sachdev, P. (1992). Golfers' Cramp: Clinical Characteristics and Evidence against It Being an Anxiety Disorder. Movement Disorders, 7, 326-332. https://doi.org/10.1002/mds.870070405

Sato, T. (2006). Development, Change or Transformation: How Can Psychology Conceive and Depict Professional Identify Construction. European Journal of School Psychology, 4, 319-332.

Sato, T. (2009). Qualitative Research Starting with TEM: Towards Research Addressing Time and Process. Tokyo: Seishin-shobou.

Sato, T., \& Tanimura, H. (2016). The Trajectory Equifinality Model (TEM). As a General Tool for Understanding Human Life Course within Irreversible Time. In Making of the Future: The Trajectory Equifinality Approach in Cultural Psychology (pp. 21-42). Greenwich: IAP Information Age Publishing, Inc.

Sato, T., \& Valsiner, J. (2010). Time in Life and Life in Time: Between Experiencing and 
Accounting. Ritsumeikan Journal of Human Sciences, 20, 79-92.

Sato, T., Yasuda, Y., Kido, A., Arakawa, A., Mizoguchi, A., \& Valsiner, J. (2007). Sampling Reconsidered: Idiographic Science and the Analysis of Personal Life Trajectories. In The Cambridge Handbook of Sociocultural Psychology (pp. 82-108). New York: Cambridge University Press. https://doi.org/10.1017/CBO9780511611162.007

Sato, T., Yasuda, Y., Kido, A., Takada, S., \& Valsiner, J. (2006). The Discovery of Trajectory Equifinality Model. Japanese Journal of Qualitative Psychology, 5, 255-276.

Schaefer, J. A., \& Moos, R. H. (1992). Life Crises and Personal Growth. In B. N. Carpenter (Ed.), Personal Coping: Theory, Research, and Application (pp. 149-170). Westport, CT: Praeger/Greenwood.

Smith, A. M., Adler, C. H., Crews, D., Wharen, R. E., Laskowski, E. R., Barnes, K., Sorenson, M. C. et al. (2003). The "Yips" in Golf. Sports Medicine, 33, 13-31. https://doi.org/10.2165/00007256-200333010-00002

Smith, A. M., Malo, S. A., Laskowski, E. R., Sabick, M., Cooney, W. P., Finnie, S. B., Kaufman, K. et al. (2000). A Multidisciplinary Study of the "Yips" Phenomenon in Golf. Sports Medicine, 30, 423-437. https://doi.org/10.2165/00007256-200030060-00004

Sugiura, T. (2001). The Empirical Study of Mental Maturity as an Athlete. Japan Journal of Physical Education, Health and Sport Sciences, 46, 337-351. https://doi.org/10.5432/jjpehss.KJ00003390626

Sugiura, T. (2004). A Narrative Study of Mental Development Process of Athletes through Their Experiences of Turning Points. Japanese Journal of Sport Psychology, 31, 23-34.

Suls, J., David, J. P., \& Harvey, J. H. (1996). Personality and Coping: Three Generations of Research. Journal of Personality, 64, 711-735. https://doi.org/10.1111/j.1467-6494.1996.tb00942.x

Suzuki, A., \& Nakagomi, S. (2013). Change of Social Support Sought by Injured Athletes during Rehabilitation. Japanese Journal of Sport Psychology, 40, 139-152. https://doi.org/10.4146/jjspopsy.2013-1129

Taku, K. (2016). Potential and Challenges of PTG. Tokyo: Kaneko Shobo.

Tamminen, K. A., Holt, N. L., \& Neely, K. C. (2013). Exploring Adversity and the Potential for Growth among Elite Female Athletes. Psychology of Sport and Exercise, 14, 28-36. https://doi.org/10.1016/j.psychsport.2012.07.002

Tamminen, K. A., \& Neely, K. C. (2016). Positive Growth in Sport. In N. L. Holt (Ed.), Positive Youth Development through Sport (2nd ed., pp. 193-204). London: Routledge. https://doi.org/10.4324/9781315709499-16

Thompson, S. C. (1985). Finding Positive Meaning in a Stressful Event and Coping. Basic and Applied Social Psychology, 6, 279-295. https://doi.org/10.1207/s15324834basp0604_1

Tominaga, S., Toyoda, N., \& Fukui, K. (2015). Qualitative Investigation of the Process of Ability Development in Performance. Japanese Journal of Sport Psychology, 42, 51-65. https://doi.org/10.4146/jjspopsy.2015-1415

Treynor, W., Gonzalez, R., \& Nolen-Hoeksema, S. (2003). Rumination Reconsidered: A Psychometric Analysis. Cognitive Therapy and Research, 27, 247-259. https://doi.org/10.1023/A:1023910315561

Tsai, J., El-Gabalawy, R., Sledge, W. H., Southwick, S. M., \& Pietrzak, R. H. (2015). Post-Traumatic Growth among Veterans in the USA: Results from the National Health and Resilience in Veterans Study. Psychological Medicine, 45, 165-179.

https://doi.org/10.1017/S0033291714001202 
Ueda, H. (2013). Daycare Teachers' Behavior and Children's Troubles: Focusing on Teaching Styles Based on the Three Layers Model of Genesis. Studies of Education of Young Children, 22, 19-29.

Valsiner, J. (2000). Culture and Human Development. Thousand Oaks, CA: SAGE Publications Ltd.

Valsiner, J. (2011). Constructing the Vanishing Present between the Future and the Past. Infancia y Aprendizaje, 34, 141-150. https://doi.org/10.1174/021037011795377601

Valsiner, J., \& Sato, T. (2006). Historically Structured Sampling (HSS): How Can Psychology's Methodology Become Tuned in to the Reality of the Historical Nature of Cultural Psychology. In Straub, Kolbl, Weidemann, \& Zielke (Eds.), Pursuit of Meaning (pp. 215-251). Advances in Cultural and Cross-Cultural Psychology, Bielefeld: Transcript.

Wada, M. (2016). Siblings of Hikikomori Youth and the Sociocultural Influences That Support and Discourage Their Autonomy from the Family. Japanese Journal of Developmental Psychology, 27, 47-58.

Wadey, R., Clark, S., Podlog, L., \& McCullough, D. (2013). Coaches' Perceptions of Athletes' Stress-Related Growth following Sport Injury. Psychology of Sport and Exercise, 14, 125-135. https://doi.org/10.1016/j.psychsport.2012.08.004

Wadey, R., Evans, L., Evans, K., \& Mitchell, I. (2011). Perceived Benefits Following Sport Injury: A Qualitative Examination of Their Antecedents and Underlying Mechanisms. Journal of Applied Sport Psychology, 23, 142-158. https://doi.org/10.1080/10413200.2010.543119

Watzlawik, M., \& Kullasepp, K. (2016). Career as Affective Journey: How Constant Flux Challenges the Search for Career Pathways and Counseling. Integrative Psychological and Behavioral Science, 50, 492-506. https://doi.org/10.1007/s12124-016-9349-3

Yamada, Y. (2007). Method of Qualitative Psychology: Listening to Narrative. Tokyo: Shinyousha.

Yasuda, Y., Arakawa, A., Takada, S., Kido, A., \& Sato, T. (2008). Young Women's Abortion Experiences: The Influences of Social Expectation and Relationships with Others. Japanese Journal of Qualitative Psychology, 7, 181-203.

Yasuda, Y., \& Sato, T. (2012). Trajectory Equifinality Model. Tokyo: Sehishin-shobou.

Zittoun, T., \& Valsiner, J. (2016). Imagining the Past and Remembering the Future: How the Unreal Defines the Real. In T. Sato, N. Mori, \& J. Valsiner (Eds.), Making of the Future: The Trajectory Equifinality Approach in Cultural Psychology (pp. 3-19). Greenwich, CT: IAP-Information Age Publishing, Inc. 